\title{
Natural Remidies at Home to Cure Complex Health Problems in Female Humans
}

\author{
Christopher J Catanzaro ${ }^{1}$, Kameswari LP ${ }^{2}$, Bhavani SVP ${ }^{3}$ and Satya SSN ${ }^{1 *}$ \\ ${ }^{1}$ Department of Agriculture, Virginia State University, Petersburg, Virginia, USA \\ ${ }^{2}$ Andhra Pradesh Horticultural University, V.R. Gudem, W.G.Dt., AndhraPradesh, India \\ ${ }^{3}$ Andhra Pradesh Agricultural University, Rajahmundry, E.G.Dt., Andhra Pradesh, India \\ *Corresponding Author: Satya Narina, Department of Agriculture, Virginia State University, Petersburg, Virginia, USA.
}

Received: August 20, 2019; Published: August 29, 2019

DOI: $10.31080 /$ ASNH.2019.03.0429

\begin{abstract}
The major health related problems like cancer, heart diseases, diabetes or obesity that women facing today are mainly due to extreme stress. The stress is connected to unemployment, family income, increase in expenditure due to increased responsibilities, and extreme work loads leading to bad food habbits, inferior quality food, and improper timings of food. Proper planning of indoor and outdoor activities will help in keeping up women's health by improved nutrition, increased less quantity meals with good quality food, food from fresh garden produce, raising flower gardens and thus achieving success in reducing stress attacks and complex health disorders.

Keywords: Factors; Women; Activity; Nutrition; Stress
\end{abstract}

\section{Introduction}

Majority of diseases in both men and women are mainly due to a lot of stress (13\%), high blood pressure (25\%), high cholesterol (20\%), diabetes $(11 \%)$ and obesity (8\%) while the rest (33\%) due to genetic or contagious or seasonal or local diseases [1]. It is mainly because of not understanding where they stand in terms of quality food intake, what their strengths are in maintaining quality starting from home to global standards and where their strengths are hidden in terms of creating employment, spending time, and money for quality of work with good quality team efforts with division of labor to relieve from their stresses or pressures from the current day curriculum starting from women in our simple nuclear families at home to state, national or internationally reputed companies and higher education or research organisations.

On an average, based on the research studies, it was observed that human females are more intelligent, challenging compared to human males in all sectors of employment. Human males though smart in getting livelihood, but are more stressed being family head compared to females. Women are less smarter to succeed though achieves higher education on par with males. There are several factors that hinder the growth of a human female whether she is staying at home as a house wife or working, whether she is educated or not, whether she is married or not or whether she is in developed or developing country.

Some of the major contributing factors were listed after reviewing literature, available as of August 19th 2019, from various online journals and reports of brief surveys conducted by food re- search groups of United States Department of Agriculture - Agricultural Research Station at Beltville (USDA-ARS) in Maryland and International food information council foundation [1] in different communities living in developed and developing countries.

\section{Major factors of concern for complex health problems}

The main factor hindering her growth is herself, because one's growth depends on their own involvement and continuous struggle for achieving success in everything they do and can be possible if women try. The only challenge a woman has by birth is being strong in every encounter at home or work. But the strength comes from various factors as described below that she has been living with, besides education being the major factor of strength to stand in any society.

\section{Nutritional and health values of consumed food}

People often complain by comparing their health problems with the amount or number of food items they consumed. The number of meals are not important when compared to the quality and amount of the quality food taken in. The quantity of inferior quality food is directly proportional to percent risk due to complex diseases of heart, liver, stomach or obesiety problems in women. Women are more inclined to eating rotten food thinking of unnecessary "SAVING" concepts at home from ages despite of their economic status in a society. On an average the amount of food taken by average female head in a family is 6 to 10 full plates (2 plates per meal/ day) of carbohydrate rich food with little greens and more spices in developing countries while in developed countries it is exactly the opposite with little quality food not enough to balance their entire 
body. Both are having dangerous impact on health as they are not balanced in their ultimate survival health values.

The food we consume today is 100 percent processed including staples foods. For example brown rice is healthier than polished white rice, and it provides minerals, vitamins, bioactive compounds besides calories in the form of carbohydrates, fat and fibre. But people are consuming more polished white rice due to its palatability, shelf life and easy cooking ability. Polished rice has a higher glycemic load and may impact glucose homeostasis inside the human body. It can be considered part of a "healthy" plate only when combined with other foods. Intake of brown rice is more viable to face the future challenges in climate change and population growth to have sustainability, adequate supply, and nutritional integrity [2].

Though intake of brown rice reduces the quantity of polished rice consumption, but equal fortification with other foods like leafy greens, beans, pulses, fruit vegetables, nuts and fruits is necessary for balanced nutrition of a human. For example take consumption of rice, brown rice and wheat as present day consumed staples by majority of global population, all are having equal values of potential nutrients. The quantity always was playing major role in reaching satiety values in developing countries unlike modern developed communities of women due to the climate and physical work they have to do every day for which fortification with other foods is always necessary to eliminate malnutrition and for good health. When consider the women in developed countries, elimination of malnutrition is possible only by increasing the number of meals per day. Women health can be improved only by replacing their heavy loads of staple cereal intake by fresh fruits and raw or steamed vegetables no matter where they live and to elimnate the extra sodium unnecessary for human body.

A survey conducted for sodium and related nutrients (energy, potassium, total and saturated fat, and total sugar) in $125 \mathrm{popu}-$ lar, sodium-contributing, commercially processed and restaurant foods with added sodium. The results revealed no changes in majority of the label sodium levels of Sentinel Foods analysed except $18.6 \%$ foods with significant changes with high sodium content and inconsistent variability in related nutrients under laboratory analysis. Continued efforts are needed by the food manufacturers to lower the sodium content of packaged and restaurant foods, and for public health officials to monitor the progress in reducing the sodium levels in food supplied [3].

Another food survey during 2009-2014 by What we Eat in America (WWEIS) and National Health and Nutrition Examination Survey (NHANES) team at USDA affiliated with George Mason University research group revealed that there is possibility of 0.35 $\%$ (one in 283) incidence of undiagnosed and untreated celiac disease in adults. This is a gastrointestinal, malabsorptive condition leading to malnutrition and osteoporosis with reduced bone health occurring due to consumption of higher amounts of calcium and phosphorus through their diet [4].

\section{Flexible food and behavioural habits}

Bad food habbits like eating high cholesterol foods due to taste, drinking lot of coffee and tea which contains unhealthy activators of neural/brain cell functions and if consumed with fortified milk (with lot of calcium and other vitamins) accumulates unnecessary load of minerals and vitamins causing toxicity to the healthy cells in organ systems of a human body leading to heart attack, urenary/ liver and lung disorders. Bad habbits are also developed due to low economic status which lead to low purchasing power of costly fresh and good quality produce. Common practice of purchasing less costly produce is observed in average income families of below $\$ 35,000$ per annuum.

Due to globalisation, human were also facing challenges in adapting the habits of new location where finding a food from home country or location is a difficult and costly task. This makes difficulty in survival, a most commonly observed feature in females compared to males. If you take the example of a vegetable "Indian spinach", it can grow as pole type in cold climates and as vine type in tropical climates depending on its adaptation and acclimatisation in accessing nutrients required from the provider "Earth" for its healthy growth. Thus adaptation is the key factor controlling women health

The availability of preferred food is always a necessity for women though quality of preferred food is always at higher hierarchy for good health. Quality is achieved with undivided attention to prevent escape of nutrients off from produce while cooking carefully. For example cooking is required for certain vegetables in certain locations of globe, but steaming vegetables is sufficient in certain other locations.

A survey of 1009 Americans between ages of 18 and 80 [1] revealed the secrets of link between food, purchasing power, consumer's confusion in accepting certain goods (Ex: cost, source, GMOs origin or freshness or taste), food insecurity and desired health outcomes. This report also stated lack of awareness among some customers about the new products or fresh foods in the market, some are scared to purchase new product, distrust in the private agencies that produce the goods with pesticide residues. Thus familiarity is playing crucial role in food marketability. The government agencies and health care providers are the most trusted in choosing the nutritious foods.

Therefore, consumers have to develop good quality dietary intake habbits and purchasers must have good quality education in choosing the right product with nutrition. Trust and better adaptative skills are necessary to protect the human race by providing fresh food with good nutrition in the entire globe when looking for sustainability, spending valuable time in the global market for choosing the right quality produce and relieving the population from long term dependence on medical supplements at later ages. 


\section{Physical activity}

Modern women are mostly confined to home, jym for physical exercises, and a computer due to technology jobs for enjoying good quality life indoors. Not even have time to breath fresh air outside their home and no time to spend little time in raising kitchen garden to have their fresh veggies organically grown in their backyard when season and time permits wherever they are located in this entire globe. If you take the examples of women who lived actively until 1980's, and of course some are still living who are in their sixties were more into kitchen gardening, raising their chickens or goats in their backyard and caring their families by providing home grown good quality food. Further this activity physically keeping them active, covering their leisure time in healthy atmosphere outside and increasing their living age.

Women can be happy by doing small healthy physical activities in and around their house to keep them stress free and their families away from a lot of health related diseases. It is advisable to reduce the marathon or running for more than what is required to reduce unknown health disorders in women. We are writing this based on evidence based results in health of women who suffered for a long period after adopting this $5 \mathrm{~K}$ to $100 \mathrm{~K}$ runs on threadmill. Readers can see the websites for women's physical exercises https://www.womensrunning.com/ which were not at all worth doing if concerned about long term health. Just like over eating tasty food leads to obesity and diabetes, physical activity beyond the basic requirement leads to health disorders especially in women. A simple plan of taking care of household activities will keep anyone healthy.

\section{Stress factor reduction}

People are adopting to yoga, walking (marathon, jogging) and other means of physical exercises in modern world in response to fighting with their unknown stress problems they face everyday and saying this physical activity will reduce stress. Actually a physical activity will increase food intake, making some people addicted to food or human machines to do lot of work beyond their limitation per day and increases their stress levels internally over the period, though successful in the outside world for a short term. Over all reducing their living age and happy life.

The most important factor to reduce alomost $90 \%$ stress in a family or a community you are associated with is keeping yourself calm, learning the habbit of being silent in all the situations and participating in team work. Team based approach gives 100 per cent results in stress reduction and longivity. This factor will give 100 percent rewards too to your happiness with good health without any problems related to neurology, blood pressure, cholesterol, cancer, diabetes, bone pains or any health related disorders.

\section{Societal and community involvement}

Twenty two percent of population are living with a family income of below $\$ 35,000$. In a family, female plays the key role in putting up with family standards through her quality contribution for nutrition. In a society, a community based kitchen garden would help families living in that community to feed quality greens and veggies instead of raising ladies clubs for playing games, physical exercises, marathons, swimming pools to entertain kids or themselves. This community based activities not only involve young generation in learning to know what they eat but also engage them in a healthy activity in a healthy fresh uncontaminated open climate. For this to happen, a massive female force has to take intitiative by sparing some of their time from the above mentioned daily activities towards raising fresh garden produce and cooking fresh food to build future generations besides educating them with high standards.

As per IFICF, the cost of fresh fruits and vegetables is high in todays market which delimits the customer to purchase those and the humans at the age of 35 or below (31\%) are living in majority in this group compared to old age group (18\%). Some of the plants in the garden if replaced with fruits and veggies of nutritive value by small simple efforts of every human female of every family in this world can lead to healthy families of future generations who can feed their families with organic produce free of pesticides.

A community based flower garden also helps to give happiness, reduce stress by active participation and spread aesthetic values to everyone who visit including women at all ages when they spend their time atleast few minutes to an hour in that garden. The flowers can be useful to extract scents, pray GOD, useful for community during festivals and other grand parties and raise income for caring people in the community during emergency.

\section{Education}

Education is not the primary factor to effect the health of a women in modern world, because more than $75 \%$ of americans or global population is educated beyond graduation from a professional organisation. Among these, per cent women are highest who are staying at home too facing all these problems unlike uneducated housewives who are stress free all the time. Women education has negative impact on her health by increasing stress levels. The reasons might be the unemployment, necessities, family income, societal or educational status or fame, the goals that they set during their education from childhood, or dreams to full fil or community service or other unknown health reasons. If the sress is in educated women due to unemployment, it is better to travel places and see the beauty of the world to reduce internal stress.

This kind of stress only be solved by their active involvement with family activities, in raising their kids with standards that are required for their needs and wishes, in trying to achieve their goals, trying to do the basic stress free activities at home as a team and participating in community gatherings for women empowerment through which one can use their knowledge to provide some service to agencies, just as explained to rasie a community garden for fresh greens, in return can get some satsifactory income and stress free life by give and take concept through team efforts. A good team is a happy home just like a good book. 
Gathering with friends, relatives and family members for fun

More than $50 \%$ women think gathering with friends, family, and relatives will reduce their stress or gives more fun than living for their family and sharing their family stresses. Some of the gatherings might provide a relief from stress, but some increase the stress. It is the choice of a women which ones to encourage in their life time and the ones to avoid forever.

Men are devoted to their family responsibilities too in modern world unlike 1940 's or ancient world because women are also doing outside distant jobs. If men are dominating even at home in caring family members besides their busy jobs at work and outside, replacing a primary duty of women at home from ages, indicates educated humanity is going backward than moving forward, reduction in primary family values and increasing stress levels of entire family. Therefore, a happy family at home leaves women 100 percent stress free. Instead of searching for fun outside, doing stress free exercises, devotion of women time for providing happiness to their loved family members will reduce the stress forever.

\section{Pscychological distress and other factors hindering a} women's freedom to go outside the home

Last but not least, anyone can be healthy and happy when they never forget to keep up with their friends, relatives and family. Now a days due to nuclear families, the distant jobs keeping the families far from each other and creating a slow raise in the health related issues and stress. These stresses of psychological base can be eliminated only by frequent visits to family, friends or relatives and may be helpful to reduce some stress [5]

Many women complain about the factor "freedom". Psychologically proved that the stress usually reduces with freedom, as humans are free living animals in a society. But the freedom to a human comes from the maturity and internal mindset not from roaming outside without an aim and freedom comes with a sincere responsibility when living outside from a family or home. Whether working women or house wives, the enitre freedom lies within them, but not with their friends or family. Freedom is there in everywork a women or men does in their daily lives with their own choice to make them happy living humans.

\section{Conclusion}

A women in this modern world can be happy in many ways if she choses the right path that fits her circumstances, for the happy family she has been living for and with a solid responsibility by following the basic principles of life and work balance with a priority to limitations of the family set boundaries.

\section{Acknowledgements}

Authors like to thank Mr. Pradeep Kumar Sripathi for providing computer and highspeed internet facility to search available literature and successfully complete this review article on time.

\section{Bibliography}

1. IFICF. International food information council foundation. Executive summary and key findings from 2018 Food and Health Survey (2018): 5-6.

2. Naomi F and Lewis Z. "Rice: Importance for Global Nutrition. Part of USDA Research Project on "Elucidating phytonutrient bioavailability, health promoting effects and mechanisms of existing/emerging foods and Beverages". Publication type: Proceedings". Journal of Nutritional Science and Vitaminology (2019).

3. Ahuja JK., et al. "Assessing Changes in Sodium Content of Selected Popular Commercially Processed and Restaurant Foods: Results from the USDA: CDC Sentinel Foods Surveillance Program". Nutrients 11.8 (2019): 1754

4. Lara S., et al. "Nutritional intake and bone health among adults with probable undiagnosed, untreated celiac disease: What we Eat in America and NHANES 2009-2014. Part of research project on "What we Eat in America - Dietary Survey: Data Collection, Interpretation, Dissemination, and Methodology". Publication type: Proceedings". Journal of American College of Nutrition (2019).

5. Wu Y., et al. "Interactions between Food and Gut Microbiota: Impact on Human Health". Annual Review of Food Science and Technology 10 (2019): 389-408.

Volume 3 Issue 9 September 2019

(c) All rights are reserved by Satya SSN., et al. 